\title{
Study on the trends in Korean clinical practice guidelines development
}

\author{
So-Youn An ${ }^{1}$, Hyun Jeong Kim², Seungoh Kim³, Jongbin Kim², Kwang-Suk Seo ${ }^{2}$, Deok-Won Lee ${ }^{5}$, \\ Kyung-Gyun Hwang ${ }^{6}$ \\ 'Department of Pediatric dentistry, College of Dentistry, Wonkwang University, Daejeon, Korea, \\ 2Department of Dental Anesthesiology, School of Dentistry, Seoul National University, Seoul, Korea, \\ ${ }^{3}$ Department of Dental Anesthesiology, School of Dentistry, Dankook University, Cheonan, Korea, \\ ${ }^{4}$ Department of Pediatric Dentistry, School of Dentistry, Dankook University, Cheonan, Korea, \\ ${ }^{5}$ Department of Oral and Maxillofacial Surgery, Kyung Hee University Dental Hospital at Gangdong, Kyung Hee University, Seoul, Korea, \\ ${ }^{6}$ Department of Dentistry/Oral \& Maxillofacial Surgery, College of Medicine, Hanyang University, Seoul, Korea
}

\begin{abstract}
Background: The definition of evidence-based clinical practice guidelines (CPGs) is "statements that are systematically developed to assist in the doctors' and patients' decision-making in certain situations." This study aims to establish the concept of evidence-based CPGs and investigate the development status to seek measures to apply evidence-based methods to CPG development for dental sedation in Korea.

Methods: The study conducted systematic searching methods based on evidence-based CPGs. Articles published between 1995 to 2015 were searched on a Korean database and the international database PubMed. The search was based on keywords related to four subjects (dentistry, clinical practice, guideline, recommendation). Two authors independently reviewed the searched articles to determine their analysis inclusion and the convergence stages, and to arrive at a conclusion through discussion.

Results: A total of 65 Korean CPGs were included. There were 51 medical guidelines, of which seven were dental and seven were Oriental medicine.

Conclusions: As a basic direction for the development of evidence-based CPGs, this work suggests the following: increased awareness; consensus on the need to supply evidence-based development methods; education, computerization, and systematic observation of evidence-based CPG development methods; continuous research development and distribution of CPGs; and creation of a database for Korean clinical dentistry practice outcomes
\end{abstract}

Key Words: Clinical practice; Dentistry; Evidence-based; Guidelines

\section{INTRODUCTION}

The American Dental Association defines evidencebased dentistry as "an approach to oral healthcare that requires the judicious integration of systematic assessments of clinically relevant scientific evidence, relating to the patient's oral and medical condition and history, with the dentist's clinical expertise and the patient's treatment needs and preferences" [1]. Clinical practice guidelines (CPGs) can be defined as "statements that are systematically developed to assist doctors' and patients' decision-making in certain situations" [2]. These guidelines maintain treatment sustainability and are regarded as effective tools used to reduce the gap between the doctor's treatment and scientific evidence. In the last 20 years, CPGs have been increasingly utilized to improve the quality of healthcare worldwide. The fundamental purpose of CPGs is to provide help for the doctors' and patients' decision-making processes with regard to
Copyright(C) 2016 Journal of Dental Anesthesia and Pain Medicine
Received: 2015. December. 29. - Revised: 2016. January. 28. Accepted: 2016. March. 9. Corresponding Author: Kyung-Gyun Hwang, Department of Dentistry/Oral \& Maxillofacial Surgery, College of Medicine, Hanyang University, 222 Wangsimni-Ro, Seongdong-Ku, Seoul 133-792, Korea Tel: +82-2-2290-8676 E-mail: hkg@hanyang.ac.kr, hkg@hmc.hanyang.ac.kr 
treatments. The guidelines use scientific evidence to demonstrate the mediation benefits and guide the doctors in determining available effective treatment methods by providing data that support such suggestions. Meanwhile, patients are informed of the treatment risks. CPGs provide an objective image of the result possibilities while clearly stating the recommendations to the doctors and providing the most up-to-date information. Moreover, the guidelines can provide information on the best treatment, thereby increasing compliance with the treatment and allowing patients to make informed decisions [3,4].

The fundamental background for the CPG increase includes the rise of uncertainty with regard to the effects of new technologies due to new medical techniques being quickly implemented. Treatments vary greatly in terms of operation, hospitalization, and drug use rates. However, the scientific base with which to explain them is inadequate. Further, there is an increasing need to encourage healthcare providers to cover a proper range of medical treatments, as doctors have a considerable amount of discretion in healthcare expenses [5].

CPGs began to emerge approximately a century ago, when doctors gathered their opinions based on their personal experiences or those of medical offices in each individual hospital; these accounts later evolved into proper literature. Currently, methods for developing CPGs can be divided into inducement of agreement and evidence-based approaches. Until the 1980s, the primary method used was inducement of agreement, in which experts gathered for a specific topic to derive treatment recommendations through discussion and agreement. This method often included the experts' own treatment methods that they had been practicing for years as is, even though their effects were not verified. Thus, this method could lead to arbitrary recommendations that may be difficult to expand, thereby stirring controversy on the consensus opinion's validity [6].

An evidence-based approach was developed to supplement the absence of explicit standards and methodologies in the former method. This new approach was developed along with the advancement of evidence- based medical methods. In other words, bibliographic search software was introduced to examine systematic literature and remove errors in the evidence selection process. Moreover, the increase in meta-analyses conducted and the creation of influential data sources, such as the Cochrane database, facilitated the organization of the superior evidence. Consequently, recommendations are formulated after more convincing processes. At present, an evidence-based development method is used more because of the objective literature collection, evaluation, and recommendation deductions advantages [7-9].

This study aims to establish the evidence-based clinical practice guidelines concept (CPGs) and investigate the development status to seek measures to apply evidence-based methods to the CPG development for dental sedation in Korea.

\section{MATERIALS AND METHODS}

\section{Literature search in databases related to the main CPGs}

The study investigates systematic searching methods based on evidence-based CPGs. Articles published between 1995 to 2015 on KoreaMed, the Korea Institute of Science and Technology Information (KISTI), DBpia, the Korea National Assembly Library, the Korean Studies Information Service System (KISS) were searched on the Korean database. Articles from the same time frame were also searched on the international database PubMed based on three search term combinations: (dentistry OR dental health services OR dental clinics OR dental offices OR dental) AND (clinical practice guidelines [ptyp] OR practice guidelines as Topic [Mesh] OR practice guideline*[TI]) AND (recommendation or consensus).

Representative websites within and outside of Korea that were related to main clinical practice guidelines were searched (Tables 1, 2). Two authors independently reviewed searched articles to determine analysis in- 
Table 1. Clinical practice guidelines (CPGs) websites

\begin{tabular}{ll}
\hline \multicolumn{1}{c}{ Name } & \\
\hline Korean Medical Guideline Information Center (KOMGI) & http://www.guideline.or.kr/ \\
Korean Guideline Clearinghouse & http://www.cpg.or.kr/ \\
GIN International Guideline Library & http://www.g-i-n.net/library/international-guidelines-library \\
AHRO National Guideline Clearinghouse & $h \mathrm{htp} / /$ www.ahrq.gov/ \\
National Institute for Health and Care Excellence (NICE) & $\mathrm{http://www.nice.org.uk/guidance}$ \\
Scottish Intercollegiate Guideline Network (SIGN) & $\mathrm{http} / /$ www.sign.ac.uk/ \\
National Health and Medical Research Council (NHMRC) & http://www.nhmrc.gov.au/guidelines-publications/how-nhmrc-develops-its-guidelines \\
\hline
\end{tabular}

Table 2. Evidence-based dentistry journals

\begin{tabular}{ll}
\hline Journal Title & Website \\
\hline Evidence-based Dentistry & http://www.nature.com/ebd/index.html \\
Journal of Evidence-based Dental Practice & http://journals.elsevierhealth.com/periodicals/ymed/home \\
\hline
\end{tabular}

Table 3. Summary of developed guidelines in Korea

\begin{tabular}{|c|c|c|c|c|c|}
\hline & Name & Year & $\mathrm{PICO}$ & Opinion & Version \\
\hline \multicolumn{6}{|c|}{ Affiliated under the Korean Academy of Dental Sciences } \\
\hline 1 & Pediatric dental sedation guideline & 2004 & & 0 & $1 \mathrm{st}$ \\
\hline 2 & Clinical pediatric dental treatment guideline & 2008 & & 0 & $1 \mathrm{st}$ \\
\hline 3 & Dental sedation guideline & 2010 & & 0 & $1 \mathrm{st}$ \\
\hline 4 & Dental implant guideline & 2010 & & 0 & $1 \mathrm{st}$ \\
\hline 5 & Infection management guideline & 2010 & & 0 & $1 \mathrm{st}$ \\
\hline 6 & Dental smoking cessation guide book & 2011 & & 0 & $1 \mathrm{st}$ \\
\hline 7 & Manual for family dentists of children and the youth & 2012 & & 0 & $1 \mathrm{st}$ \\
\hline \multicolumn{6}{|c|}{ Korean Institute of Oriental Medicine affiliated under the Society of Korean Medicine } \\
\hline 1 & Acupuncture CPG for musculoskeletal symptoms (low back, neck, knee pain) & 2013 & & 0 & $1 \mathrm{st}$ \\
\hline 2 & CPG of Hwa-Byung & 2013 & & 0 & $1 \mathrm{st}$ \\
\hline 3 & Korean medicinal CPG for facial nerve palsy & 2014 & & 0 & $1 \mathrm{st}$ \\
\hline 4 & Atopic dermatitis Korean medicine CPG & 2014 & & 0 & $1 \mathrm{st}$ \\
\hline 5 & Korean medicine CPG for herniation of lumbar disk & 2014 & & 0 & $1 \mathrm{st}$ \\
\hline 6 & Korean medicine CPG for ankle sprain & 2015 & & 0 & $1 \mathrm{st}$ \\
\hline 7 & Korean medicine CPG for shoulder pain & 2015 & & 0 & $1 \mathrm{st}$ \\
\hline \multicolumn{6}{|c|}{ CPGs affiliated under the Korean Academy of Medical Services } \\
\hline 1 & Guideline for the drug treatment of epilepsy & 2001 & & 0 & $1 \mathrm{st}$ \\
\hline 2 & Guideline for allergy rhinitis treatment & 2003 & & 0 & $1 \mathrm{st}$ \\
\hline 3 & Medical guideline for bronchial asthma & 2005 & & 0 & $1 \mathrm{st}$ \\
\hline 4 & Guideline for rhinosinusitis treatment & 2005 & & 0 & $1 \mathrm{st}$ \\
\hline 5 & Guideline for the treatment of adult community pneumonia & 2005 & & 0 & $1 \mathrm{st}$ \\
\hline 6 & $\begin{array}{l}\text { Standard guideline for the categorization of operations on chronic otitis media and reporting the } \\
\text { result after surgery }\end{array}$ & 2006 & & 0 & 1 st \\
\hline 7 & Guideline for the treatment of asthma & 2006 & & 0 & $1 \mathrm{st}$ \\
\hline 8 & Guideline for the treatment of diabetes & 2007 & & 0 & $1 \mathrm{st}$ \\
\hline 9 & Latest views on hormonal therapy & 2007 & & 0 & $1 \mathrm{st}$ \\
\hline 10 & Guideline for the treatment of shingles and post-therapeutic neuralgia & 2008 & & 0 & $1 \mathrm{st}$ \\
\hline 11 & Guideline for the treatment of chronic kidney diseases 2008 & 2008 & & 0 & $1 \mathrm{st}$ \\
\hline 12 & The third breast cancer treatment recommendation & 2008 & 0 & & 3rd \\
\hline 13 & Guideline for the treatment of complex airway management & 2008 & & 0 & $1 \mathrm{st}$ \\
\hline 14 & Guideline for the treatment of post-traumatic stress disorder & 2008 & 0 & & $1 \mathrm{st}$ \\
\hline 15 & Guideline for the treatment of depression, 2008 & 2008 & & 0 & $1 \mathrm{st}$ \\
\hline 16 & Perioperative care of patients using anticoagulants & 2008 & & 0 & 1 st \\
\hline 17 & Diagnosis guideline for intestinal Behcet's disease & 2008 & & 0 & $1 \mathrm{st}$ \\
\hline 18 & Diagnosis guideline for ulcerative colitis & 2009 & & 0 & $1 \mathrm{st}$ \\
\hline 19 & Diagnosis guideline for intestinal tuberculosis & 2009 & & 0 & $1 \mathrm{st}$ \\
\hline
\end{tabular}

(Continued to the next page) 
Table 3. Continued

\begin{tabular}{|c|c|c|c|c|c|}
\hline & Name & Year & $\mathrm{PICO}$ & Opinion & Version \\
\hline 20 & Diagnosis guideline for Crohn's disease & 2009 & & 0 & 1 st \\
\hline 21 & Korean guideline for the drug treatment of generalized anxiety disorders, 2009 & 2009 & & 0 & 1 st \\
\hline 22 & 2009 treatment guideline for hepatocellular carcinoma & 2009 & & 0 & $1 \mathrm{st}$ \\
\hline 23 & Korean standard treatment guideline for stroke rehabilitation & 2009 & & 0 & 1 st \\
\hline 24 & Treatment recommendation for gynecologic cancer & 2010 & & 0 & $1 \mathrm{st}$ \\
\hline 25 & Treatment recommendation for gallbladder polyps & 2010 & 0 & & 1 st \\
\hline 26 & Treatment guideline for lung cancer & 2010 & & 0 & $1 \mathrm{st}$ \\
\hline 27 & Treatment guideline for acute facial nerve paralysis & 2010 & 0 & & $1 \mathrm{st}$ \\
\hline 28 & Treatment guideline for chronic hepatitis B & 2011 & 0 & & $3 r d$ \\
\hline 29 & Treatment guideline for asthma in Korean adults & 2012 & & 0 & $1 \mathrm{st}$ \\
\hline 30 & Intravenous infusion nursing practice guideline & 2012 & 0 & & 1 st \\
\hline 31 & Clinical practice guideline for stroke rehabilitation in Korea, 2012 & 2012 & 0 & & 2nd \\
\hline 32 & Cancer pain management guideline in Korea & 2012 & & 0 & 1 st \\
\hline 33 & Guidelines for the treatment of gastroesophageal reflux disease, 2012 & 2012 & 0 & & 1 st \\
\hline 34 & Standard treatment guidelines for stomach cancer & 2012 & 0 & & 1 st \\
\hline 35 & COPD treatment guideline, 2012 revision & 2012 & 0 & & $3 r d$ \\
\hline 36 & Treatment guideline for colorectal cancer, v. 1.0 & 2012 & 0 & & $1 \mathrm{st}$ \\
\hline 37 & Treatment guideline for peritoneal dialysis and peritonitis & 2012 & 0 & & 1 st \\
\hline 38 & Treatment guideline for bile duct stones & 2013 & 0 & & $1 \mathrm{st}$ \\
\hline 39 & Treatment guideline for acute pancreatitis & 2013 & 0 & & 1 st \\
\hline 40 & Treatment guideline for pediatric ear infections, 2014 revision & 2014 & 0 & & 2nd \\
\hline 41 & Treatment guideline for gallbladder surgery & 2014 & 0 & & $1 \mathrm{st}$ \\
\hline 42 & Interventional re-entry surgery on peripheral arteries in the legs & 2014 & 0 & & 1 st \\
\hline 43 & Management guideline for hemodialysis vascular access & 2014 & & 0 & $1 \mathrm{st}$ \\
\hline 44 & 2014 evidence-based recommendations for hypertension in primary care & 2014 & 0 & & 1 st \\
\hline 45 & 2014 evidence-based recommendations for diabetes in primary care & 2014 & 0 & & 1 st \\
\hline 46 & CPG for diabetes in Korea & 2014 & 0 & & 1 st \\
\hline 47 & COPD treatment guideline, 2014 revision & 2014 & 0 & & 4th \\
\hline 48 & Asthma treatment guideline & 2014 & 0 & & $3 r d$ \\
\hline 49 & HIV/AIDS treatment guideline revision & 2014 & 0 & & 1 st \\
\hline 50 & Treatment recommendations for benign prostatic hyperplasia & 2015 & 0 & & 1 st \\
\hline 51 & Emergency medical personnel's guidelines for first aid and transfer of burn patients & 2015 & 0 & & $1 \mathrm{st}$ \\
\hline
\end{tabular}

clusion and the convergence stages, and arrived at a conclusion through discussion.

\section{Inclusion and exclusion criteria}

The CPGs included in this study were original reports published in Korea that described interventions and provided sufficient methodological details based on evidence.

The inclusion criteria were as follows: (1) Korean language CPGs, which were produced by mainland Korea organizations, and (2) results from systematic reviews of relevant research evidence.

If the CPGs were updated, only the most recent version was assessed.

CPGs were excluded if they met any of the following criteria: systematic reviews, narrative reviews, primary studies, critical pathways, training manuals for medical doctors, textbook-like publications, guidelines for patients, editorials, foreign guideline translations, secondary or multiple publications, and short summaries.

\section{RESULTS}

A total of 65 Korean CPGs were included in the study of developed Korean guidelines. The medical guidelines were the most developed. It is a step that has been established the official site. Therefore, we reviewed 51 guidelines that were organized as clinical practice guidelines affiliated under the Korean Medical Guideline 
Table 4. Guideline development agencies in several countries

\begin{tabular}{lll}
\hline Country & Guideline development agency & Website \\
\hline UK & Scottish Intercollegiate Guideline Network (SIGN) & www.sign.ac.uk \\
France & National Institute for Health and Clinical Excellence (NICE) & www.nice.org.uk \\
Germany & Haute Autoritee de Santee (HAS) & www.has-sante.fr \\
Netherland & AEZO/AQuMed Guideline Link Collection (AGLC) & www.leitlinien.de \\
New Zealand & Dutch Institute for Healthcare Improvement (CBO) & www.cho.nl \\
US & New Zealand Guidelines Group (NZGC) & www.nzgg.org.nz \\
& National Guideline Clearinghouse (NGC) & www.guidlines.gov \\
Italy & Agency for Healthcare Research and Ouality (AHRO) & www.ahrq.gov \\
Singapore & Programma Nazionale Linee Guida (PNLG) & www.pnlg.it \\
Japan & Singapore Clinical Practice Guidelines & www.moh.gov.sg \\
\hline
\end{tabular}

Information Center (Table 3).

There were seven guidelines each for dental and Oriental medicine (Table 3).

\section{DISCUSSION}

In developed nations, the policies on practice guidelines are established, with high-quality guidelines distributed and executed on the national or regional levels. The methods for developing practice guidelines are also evolving as they are systematically examined. This progress in development methods further increases the practice guidelines' validity.

Advanced nations implement national policies with regard to practice guidelines' development, expansion, and execution. In a study comparing Europe's practice guidelines, the UK, Finland, France, Germany, Italy, and Netherlands were reported to have had national policies regarding practice guidelines since the 1990s (Table 4). These policies and approaches for practice guidelines exhibit the following similarities. First, many nations have policies connected to campaigns to improve practice guideline quality. Second, most practice guideline development programs use scientifically strict methods, leading toward evidence-based methodologies. Third, most practice guideline developments are closely connected to expansion and execution strategies.

Each advanced nation places extensive effort on not only practice guideline development, but also their ex- pansions and executions. Among the many execution strategies, the most used include educational methods, such as data provisions, discussions, public opinion, leader visits, or announcements. This is in addition to such methods as providing incentives based on evaluations and finances. Most practice guideline programs have standards with which to manage the guidelines' quality. They also operate clearing houses that supply the guidelines.

Table 3 Summary of developed guidelines in Korea (Korean Institute of Oriental Medicine affiliated under the Society of Korean Medicine)

In Korea, CPG development began approximately ten years ago, which increased the health care community's interest [10].

The Korean practice guidelines that clinical associations have developed to date have the following characteristics. First, they are not comprised of multidisciplinary development groups to develop practice guidelines. Second, the recommendations are intended to develop guidelines based on evidence. However, those making the recommendations do not actually conduct systematic observations, which is crucial in an evidencebased method. They also intend to apply the actual conditions in Korea, but often only reference experiences due to the lack of Korean studies. Third, except for in a few instances, they deduce recommendations after unofficial consensus, in which the connection between the recommendations and evidences are not stated clearly. Fourth, all practice guidelines omit detailed 
descriptions of the composition of the group responsible for the development or the development methods.

One of the challenges in developing practice guidelines is to ensure that they reflect Korea's reality. This aspect entails considering the disease's epidemiologic characteristics and observing the diagnosis and treatment method effects in the Korean healthcare environment. This challenge persists due to the extremely low number of verifications on the diagnoses and treatment methods effects. Thus, the clinical studies used as evidence are often taken from those conducted overseas, while the validity in Korea remains uncertain. Overseas clinical studies can be searched comprehensively via databases, such as PubMed, EMBASE, and the Cochrane Library, whereas no database can be used to conduct a comprehensive search for Korean literature. In most cases, a manual search is required. In the long term, a database needs to be built to in order to easily search a comprehensive Korean paper collection [11]. Evidencebased methodologies are the core principles with which to develop practice guidelines. However, few individuals in Korea have experience with them. Individuals with experience in the systematic observations used to develop guidelines need to be secured and fostered.

Research regarding practice guideline methods and professional agencies or organizations responsible for developing guidelines overseas should be organized according to the Korean conditions and resources.

Further, guidelines must be developed under reasonable and well-planned methods. If not, they will not be of much of help with actual treatments and may even yield negative impacts.

\section{CONCLUSION}

Guidelines are necessary to provide a vast range of critical and objective information on the diagnoses, benefits, and limits of available treatments. This will allow doctors on site to effectively use the guidelines to determine the treatment for each patient. Studies have suggested that guidelines can change doctors' behaviors and improve treatment results. Even in Korea, there has been an increase in guideline development. These research results should be referenced sufficiently to search for appropriate development and execution strategies for Korea. For this, government and medical community support, along with cooperation and efforts on policies, is required.

\section{REFERENCES}

1. ADA Policy on Evidence-Based Dentistry. American Dental Association. 2006. Available from http://www.ada.org/ en/about-the-ada/ada-positions-policies-and-statements/ policy-on-evidence-based-dentistry

2. Field MJ, Lohr KN (eds.). Clinical Practice Guidelines: directions for a new program. Washington DC, National Academies Press. 1990.

3. Cluzeau F, Littlejohns P, Grimshaw J, Feder G, Moran S. Development and application of a generic methodology to assess the quality of clinical guidelines. Int J Qual Health Care 1999; 11: 21-8.

4. Grol R, Dalhuijzen J, Mokkink H, Thomas S, Veld C, Rutten G. Attributes of clinical guidelines that influence use of guidelines in general practice: observational study. BMJ 1998; 317: 858-61.

5. Lohr KN. The quality of practice guidelines and the quality of health care. In Guidelines in health care. Report of a WHO Conference. 1997.

6. Lugtenberg M, Burgers J, Westert G. Effects of evidencebased clinical practice guidelines on quality of care: a systematic review. Qual Saf Health Care 2009; 18: 385-92.

7. Nair R, Aggarwal R, Khanna D. Methods of formal consensus in classification/diagnostic criteria and guideline development. Semin Arthritis Rheum 2011; 41: 95-105

8. Rycroft-Malone J. Formal consensus: the development of a national clinical guideline. Qual Health Care 2001; 10: $238-44$.

9. Robinson N, Liu J, Lee MS. Clinical guidelines: the way for best practice. Eur J Integr Med 2014; 6: 133-4. 
10. Ahn HS, Kim HJ. Development and implementation of clinical practice guideline: current status in Korea. J Korean Med Sci 2012; 27: S55-60.

11. Medical Research Library of Brooklyn: The Evidence
Pyramid. [http://library.downstate.edu/EBM2/2100.htm] Choi et al. Implementation Science (2015) 10:104 Page 9 of 10. 